Rev Inv Vet Perú 1999; 10(1): 95-104

\title{
ENFOQUE
}

\section{INVESTIGACIÓN ALIMENTARIA PARA PRODUCCIÓN BOVINA CON ORDEÑO EN EL TRÓPICO ${ }^{1}$.}

\author{
Miguel Ara G. ${ }^{2}$, Manuel De La Torre V. ${ }^{3}$, César Reyes A. ${ }^{2}$ y Olger \\ Ramos C. ${ }^{2}$
}

\section{Abstract}

Dual purpose (beef and milk) cattle production levels in the Ucayali area are low despite of 25 years of research. Poor nutritional quality of available forage and reduced pasture production during the dry season, and low overall productivity of the herds, are the principal factors which limit the development of this activity. The Program on Feed Research in Dual Purpose Cattle Production has promoted research to alleviate these constraints. The components of this program include introduction and evaluation of forage germoplasm of higher nutritive quality which is tolerant of dry conditions, use of forage legumes, and incresasing the reproductive performance of the herds through better feeding strategies. Current and expected research results are described. Research impact at farmer level is defined as increased and more efficient milk production through the use of better quality pastures, higher productivity during the dry season, and increased reproductive performance of the farm herd.

Key words: Beef and milk production, Amazonia, Ucayali, Pucallpa

\section{Resumen}

La producción bovina con ordeño en Ucayali no tiene el desarrollo esperado a pesar de más de 25 años de investigación. La baja calidad nutritiva del forraje en oferta, la reducción de la producción forrajera durante la época seca, y las bajas productividades globales de los hatos en los fundos son las principales limitaciones que previenen el desarrollo de esta actividad. El Programa de Investigación Alimentaria para Producción Bovina con Ordeño ha diseñado investigación dirigida a resolver estas limitaciones. La búsqueda de germoplasma forrajero de alta calidad nutritiva y tolerante al estrés de humedad, la investigación en el potencial de las leguminosas forrajeras, el uso de suplementación estacional en época seca, y el manejo alimentario dirigido a una mayor eficiencia reproductiva son los componentes de la investigación planteada. Se describen los principales resultados obtenidos y esperados y su aplicación a la solución

' Parte del Programa de Investigación en Producción Animal de la EE IVITA-Pucallpa

2 UNMSM-FMV. Programa de Investigación en Producción Animal. EE IVITA-Pucallpa. E-mail: ivitapuc@electrodata.com.pe

${ }^{3}$ Actualmente Director de Pastos y Ganadería del Consorcio para el Desarrollo Sostenible de Ucayali. Email: codesu@mail.cosapidata.com.pe 
del problema. El impacto a nivel de productores es definido como una mayor y más eficiente producción de leche con pasturas de mejor calidad nutritiva, por una disminución de la caída de la oferta forrajera en época seca, y por una mayor productividad del hato.

Palabras clave: Ganadería de doble propósito, Amazonía, Ucayali y Pucallpa.

\section{Intruducción}

La investigación en producción bovina con ordeño ha sido iniciada por el Centro de Investigación IVITA en Pucallpa hace más de 25 años (IVITA, 1972). Importantes contribuciones tecnológicas como la definición del genotipo animal apropiado, manejo del ordeño, y manejo reproductivo y sanitario han permitido la existencia misma de esta actividad en Ucayali, traducida en más de 45 fundos familiares con ordeño, y una producción de la cuenca lechera del eje de la carretera Pucallpa-Lima de más de 1300 tn en 1997 (Oficina de Información Agraria, 1997).

A pesar de la contribución del IVITA y de otras instituciones en este tiempo, el estado de desarrollo económico de la actividad lechera en Ucayali es aún precario. Datos recientes muestran niveles de producción de sólo 31 por vaca en fundos. Fuera de factores externos como la etapa de violencia subversiva, y la falta de adecuadas políticas crediticias, de infraestructura y de comercialización hacia esta actividad, se tiene que la escasa adopción por parte de los productores de los componentes de manejo alimentario han prevenido el logro de niveles de producción concordantes con el potencial de la producción bovina con ordeño.

Actualmente, con el problema de violencia en la zona relativamente superado; con el potencial de mercado que representan Pucallpa y otras poblaciones de la selva, cuya demanda de productos lácteos es satisfecha solamente en $7 \%$ por la producción regional (Riesco, 1997); y con el reconocimiento de la producción animal como una de las opciones de Desarrollo Alternativo (Contradrogas, 1998), la oportunidad de impacto de nuevas opciones de manejo alimentario es inmejorable. En este sentido, este documento resume las principales limitaciones de conocimiento y tecnología que previenen niveles adecuados de productividad y producción, y describe una estrategia de investigación a mediano plazo, desarrollada por el Programa de Investigación en Producción Animal de IVITA-Pucallpa, concurrente con estas limitaciones, así como los principales resultados e impacto logrados y proyectados.

\section{Limitaciones a ser Resuellas a través de h Investigación}

En términos generales, la limitación de orden alimentario en la producción bovina con ordeño está expresada por una insuficiente biomasa forrajera y de baja calidad nutritiva (digestibilidad y contenido de proteína cruda [PC] de las pasturas en uso). Sin embargo, para una aproximación estratégica es conveniente desdoblar esta limitación en tres componentes, los cuales ocurren a nivel local (Fig. 1).

\section{Baja calidad nutritiva de la biomasa forrajera}

Brachiaria decumbens es prácticamente la única opción de germoplasma forrajero que domina el escenario de producción bovina con ordeño. Si bien esta especie muestra ventajas de adaptación al medio, la sostenibilidad de 


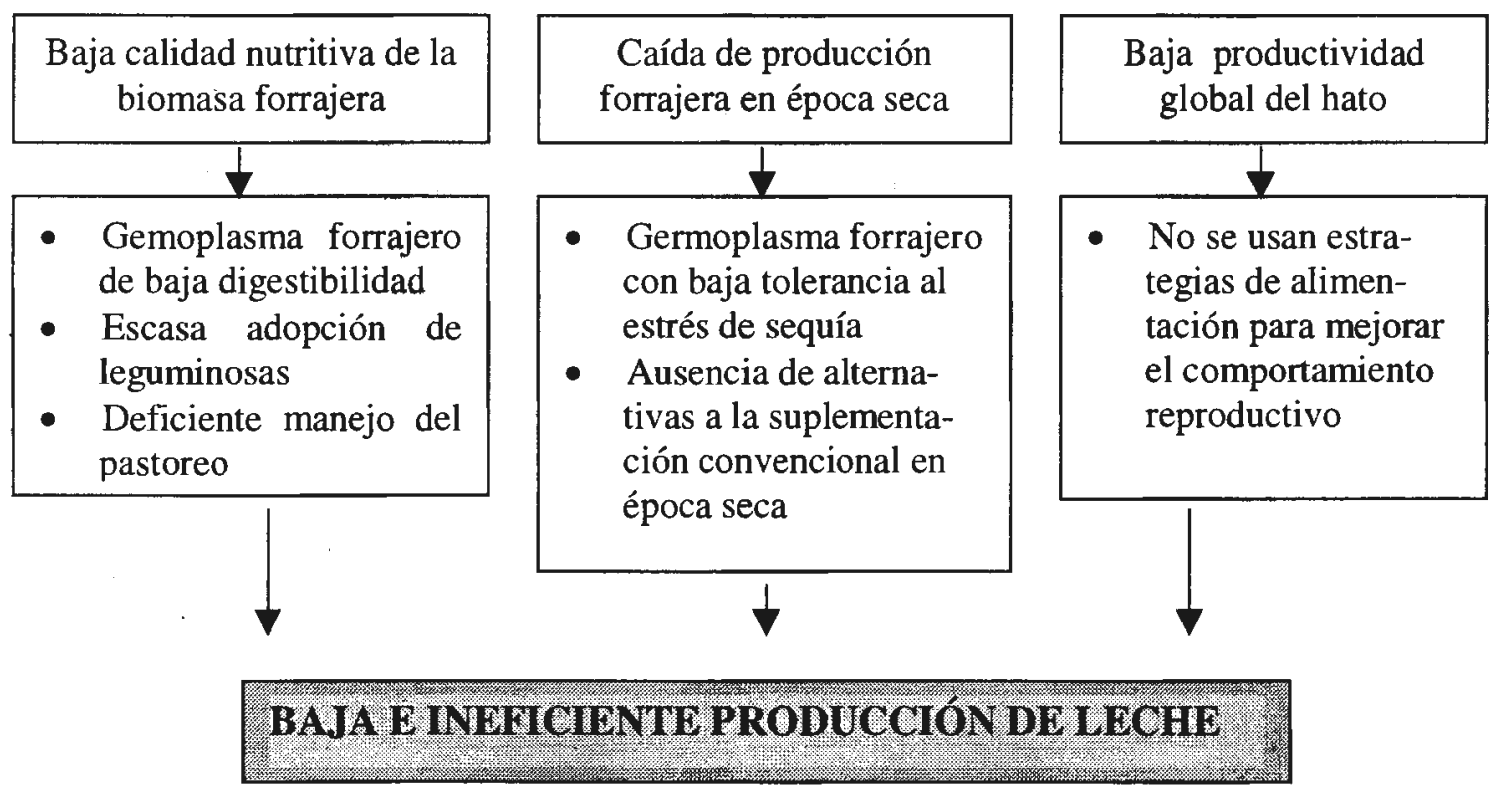

Figura 1. Componentes de la limitación de orden alimentario de la producción bovina con ordeño en Ucayali.

su producción y la capacidad de suministro de $\mathrm{PC}$ en monocultivo requiere de fertilización con nitrógeno, alternativa antieconómica en las condiciones actuales. Por otro lado, la evolución de la digestibilidad con la edad de rebrote es demasiado rápida en esta especie como para permitir un manejo flexible del pastoreo a nivel de productor. Además, $B$. decumbens tiene el problema potencial del insecto "salivazo" (Aneolamia sp., Deois incompleta, Zulia sp.) el cual ha devastado pasturas de esta especie en Colombia, Brasil y Bolivia. Todo esto hace necesario otras opciones de germoplasma de gramínea forrajeras con calidad nutritiva más alta y estable.

La adopción de leguminosas en pasturas asociadas ha sido prácticamente nula, a pesar de sus ventajas de productividad, calidad nutritiva, y suministro de proteína. El hecho de que hasta el momento no haya sido posible detectar incrementos significativos en producción de leche como resultado de la inclusión de leguminosas en pasturas en Pucallpa, ha sido señalado por varios investigadores como la principal causa de su escasa adopción (TROPILECHE, 1998). Esto sugiere la necesidad de cuantificar la contribución de las leguminosas en la producción de leche en condiciones experimentales más controladas, así como identificar indicadores metabólicos para estimar el consumo de leguminosas bajo condiciones de pastoreo.

El germoplasma forrajero adaptado a las condiciones de Pucallpa tiene una baja calidad nutritiva la cual disminuye rápidamente con la edad de rebrote. Esta situación es complicada por la tendencia de los productores a emplear bajas presiones de pastoreo y tiempos de descanso excesivamente largos. Muchos de ellos incluso permiten que $B$. decumbens entre en floración antes de pastorearlo en la creencia que así están contribuyendo a la sostenibilidad de la pastura. Aun cuando se conoce la edad óptima de rebrote para el pastoreo de $B$. decumbens, es necesario 
cuantificar este componente en términos de producción de leche.

\section{Caída de producción forrajera en época seca}

El área de influencia de IVITAPucallpa está comprendida en las formaciones ecológicas Bosque Húmedo Tropical y Bosque Estacional Semisiempreverde. Sin embargo, la intensidad y duración de la época seca son suficientes para provocar una reducción de la cantidad de biomasa de las pasturas de hasta $48 \%$ y de producción de leche de hasta $50 \%$, con la consiguiente reducción en los ingresos de los productores. Es necesario realizar Investigación dirigida a disipar esa caída en productividad, probablemente a través de la búsqueda de germoplasma forrajero tolerante a la falta de humedad.

La suplementación estratégica ha permitido aliviar la caída de productividad animal (De La Torre et al., datos no publicados), pero aún falta mayor investigación sobre el particular. Así mismo se deben evaluar otras opciones como leguminosas arbustivas (Pizarro y Coradin, 1995), las cuales pueden ser empleadas en sistemas de corte y acarreo para suplementación alimenticia durante la época seca.

\section{Deficiente oferta forrajera a vaquillas de reemplazo}

Las vaquillas destetadas son tradicionalmente relegadas en favor de las vacas lactantes en el manejo de la alimentación en sistemas de producción bovina con ordeño en Ucayali, tanto en la asignación a las pasturas de mejor calidad como en la oportunidad de suplementación alimenticia. En esta situación, el lento crecimiento de las vaquillas demora su ingreso a la etapa reproductiva y de producción láctea, disminuyendo la productividad global del hato.
Este problema es pocas veces percibido por el productor y señala la oportunidad de investigar opciones de manejo alimentario dirigido a vaquillas de reemplazo con la finalidad de que alcancen peso de empadre en el menor tiempo posible (De La Torre et al., 1998).

\section{Investigacion Necesaria}

\section{Incrementar la calidad nutritiva del forraje en oferta}

Probablemente las mejores estrategias concurrentes con este objetivo sean: (a) La búsqueda de nuevas opciones de germoplasma de gramíneas con mayor calidad nutritiva (PC y digestibilidad), (b) La cuantificación del beneficio de las leguminosas en producción de leche, y (c) Cambios en el manejo del pastoreo buscando maximizar la calidad nutritiva del forraje en oferta.

La búsqueda de nuevas opciones de germoplasma puede materializarse con la reactivación de un programa de introducción y evaluación de forrajes. Instituciones como el Centro Internacional de Agricultura Tropical (CIAT) disponen de nuevos géneros y especies con mejores características nutricionales. El contacto institucional para disponer de estas colecciones con potencial de adaptación a nuestro medio, y su establecimiento y evaluación al corte en sistemas de pequeñas parcelas (Toledo y Schultze-Kraft, 1982) en IVITA-Pucallpa son los pasos a seguir. La evaluación agronómica, obviamente, debe enfatizar la producción de biomasa, digestibilidad y contenido de PC a diferentes edades de corte.

La cuantificación del efecto de las leguminosas sobre la producción de leche debe incidir en su evaluación bajo condiciones experimentales más controladas. 
La ejecución de ensayos de alimentación de vacas lactantes en confinamiento con proporciones crecientes de las leguminosas más usadas como kudzu (Pueraria phaseoloides), stylo (Stylosanthes guianensis), y Centrosema macrocarpum debe ir paralelo a la ejecución de ensayos de pastoreo comparando gramíneas ( $B$. decumbens por ejemplo) con y sin leguminosas, en los cuales el comportamiento reproductivo de las vacas sería una variable adicional de evaluación. Los ensayos en confinamiento deben dar un control del consumo creciente de leguminosas, situación difícil de obtener en condiciones de pastoreo, y su relación con la producción y composición de la leche. La variación de índices metabólicos, como úrea en la leche, con el porcentaje de leguminosa ingerida debe permitirnos establecer relaciones útiles para predecir el consumo de leguminosas en condiciones de pastoreo. Al final, una adecuada cuantificación del beneficio de la leguminosa facilitará la adopción por los productores en el ámbito de influencia. Así mismo, es necesario complementar estos trabajos con investigación agronómica de establecimiento en leguminosas de generación relativamente nueva como $C$. macrocarpum.

La búsqueda de alternativas en el manejo del pastoreo tendientes a la optimización de la calidad nutritiva del forraje en oferta debe incluir la ejecución de ensayos de pastoreo en los que la principal variable causal sean diferentes edades de rebrote en sistemas de pastoreo rotativo con vacas lactantes. Brachiaria decumbens, o alguna de sus alternativas actualmente en uso como $B$. dictyoneura, sola o asociada puede ser la pastura en evaluación. Una relación funcional entre las diferentes edades de rebrote y la digestibilidad y contenido de PC sobre la producción de leche nos debe permitir optimizar este componente del manejo del pastoreo. Las dificultades en la obtención del área de pasturas disponibles y el costo de instalación pueden reducirse mediante el uso de disposiciones experimentales de sobre-cambio o de cuadrado latino.

\section{Reducir la caída de productividad durante la época seca}

La tolerancia del forraje a la sequía y la suplementación animal durante la época seca parecen ser dos de las estrategias más convenientes para reducir la caída de productividad durante la época seca. La investigación de soporte a la primera estrategia sería la introducción y evaluación de nuevo germoplasma forrajero, gramíneas y leguminosas con características de tolerancia al estrés de humedad. Todos los materiales con una reducción de producción de biomasa en época seca menor que $48 \%$, además de una producción razonable durante el año, deberían ser tomados en cuenta para evaluaciones posteriores.

Se ha demostrado, usando raciones comerciales, que la suplementación en época seca es tan conveniente como la suplementación permanente, especialmente para animales en crecimiento. Sin embargo, es posible aplicar la suplementación estacional con forrajes en un sistema de corte y acarreo. La investigación pertinente estaría basada en ensayos de suplementación diferencial, primero a vacas lactantes, y en una segunda fase a vaquillas de reemplazo, ambas en pastoreo. Un material promisorio para suplemento es la leguminosa cratilia (Cratylia argentea), la cual ha demostrado ya sus ventajas de productividad y calidad nutritiva en época seca (Pizarro y Coradin, 1995). Material adicional para este sistema debe ser provisto gradualmente por los ensayos de introducción y evaluación agronómica de germoplasma tolerante a sequía. 
Mejorar la oferta alimentaria en vaquillas de reemplazo

Existe oportunidad de lograr alcanzar la etapa reproductiva en menor tiempo en vaquillas de reemplazo. Debido a que tradicionalmente los productores sesgan sus recursos hacia las vacas en producción, se deben realizar, previamente a cualquier experimentación, actividades de extensión mediante visitas, charlas o seminarios. El objetivo de estas actividades es que el productor perciba que la productividad global del hato, a través, de una mejor eficiencia reproductiva, es tan importante como los niveles de rendimiento de los animales en producción. Posteriormente deben ser diseñados, en forma participativa, ensayos de alimentación en fundos en donde se contraste la alternativa de manejo alimentario dirigido a la eficiencia reproductiva $v s$. la práctica tradicional. Las opciones de manejo alimentario a ensayarse varían desde el uso de pasturas mejoradas para animales de reemplazo hasta el uso de suplementación estacional con alimentos concentrados o con forrajes en sistemas de corte y acarreo.

El Programa de Investigación en Producción Animal de IVITA-Pucallpa ha ejecutado ensayos dirigidos al logro de los objetivos mencionados anteriormente. El Cuadro 1 describe la investigación realizada o en marcha, en términos de las limitantes señaladas en la Fig. 1.

La aproximación inicial se ha dirigido a resolver el problema de adopción de leguminosas. Ensayos agronómicos con C. macrocarpum deben proporcionar componentes de la tecnología de

Cuadro 1. Investigación realizada por el IVITA dirigida a resolver las principales limitantes de orden alimentario que afectan la producción de leche en Ucayali.

\begin{tabular}{lll}
\hline \multicolumn{1}{c}{ Limitante } & \multicolumn{1}{c}{ Investigación } & Estado \\
\hline $\begin{array}{l}\text { Escasa adopción de } \\
\text { leguminosas }\end{array}$ & $\begin{array}{l}\text { Manejo del establecimiento de } C . \\
\text { Macrocarpum } \\
\text { Potencial de leguminosas en vacas } \\
\text { en ordeño en confinamiento } \\
\text { Potencial de leguminosas en vacas } \\
\text { en ordeño al pastoreo }\end{array}$ & En marcha \\
$\begin{array}{l}\text { Deficiente manejo del } \\
\text { pastoreo }\end{array}$ & $\begin{array}{l}\text { Edad óptima de rebrote para el } \\
\text { pastoreo de } B \text {. dictyoneura }\end{array}$ & En marcha \\
$\begin{array}{l}\text { Disminución de la oferta } \\
\text { forrajera en época seca }\end{array}$ & $\begin{array}{l}\text { Suplementación estacional en } \\
\text { época seca }\end{array}$ & Realizada \\
$\begin{array}{l}\text { No se usan estrategias de } \\
\text { alimentación para mejorar } \\
\text { la eficiencia reproductiva }\end{array}$ & $\begin{array}{l}\text { Suplementación mineral: niveles y } \\
\text { frecuencias }\end{array}$ & Realizada \\
\hline
\end{tabular}


establecimiento en términos de la densidad de siembra y fertilización con $\mathrm{P}$, los cuales deben facilitar la difusión de esta leguminosa considerada como la mejor alternativa a stylo. Paralelamente, se está tratando de cuantificar el potencial de las leguminosas forrajeras en sistemas con ordeño, tanto en condiciones controladas de confinamiento, como al pastoreo.

La edad óptima de rebrote para el pastoreo rotativo de $B$. dictyoneura es una de las más importantes alternativas a $B$. decumbens. Esto debe proporcionar al productor una herramienta para el manejo del pastoreo dirigido a optimizar la calidad nutritiva del forraje en oferta.

La suplementación estacional durante la época seca, comparada a la suplementación permanente debe proporcionar una opción económica para aminorar la caída de productividad animal durante este período.

La suplementación mineral, recurso caro y escaso, es actualmente aplicado en forma indiscriminada. Información sobre niveles y frecuencias adecuadas de dosificación deben permitir al productor administrar este recurso en forma más eficiente hacia el logro de mejores niveles de producción.

\section{Investigación Proyectada}

La proyección a mediano plazo es hacia el reforzamiento de la investigación en nuevas alternativas de germoplasma forrajero con ventajas de calidad nutritiva y tolerancia a sequía. Consecutivamente, las mejores opciones deben ser adecuadas al uso en las diferentes etapas de la producción bovina con ordeño, ya sea hacia el mejoramiento de los niveles de producción o del comportamiento reproductivo.
Adicionalmente se iniciará investigación en el uso de una nueva herramienta que debe facilitar el manejo animal a nivel de productores: los rangos de condición corporal. Los principales componentes de la investigación proyectada son descritos en el Cuadro 2.

\section{Resultados}

Los resultados, obtenidos o previstos, deben traducirse en componentes de tecnología dirigidos al objetivo general de aumentar la producción de leche en Ucayali. El Cuadro 3 resume los principales resultados obtenidos y esperados como producto de la esta iniciativa de investigación.

\section{Impacto}

Consistentemente con las limitaciones planteadas esquemáticamente al inicio de este documento, la Fig. 2 grafica los componentes más importantes del impacto a nivel de productores que tendrían los resultados de la investigación aquí descrita.

Necesariamente, este impacto está condicionado a la optimización de variables concomitantes, internas y externas a la actividad. Entre las variables internas están el uso del genotipo adecuado para productividad y tolerancia al estrés ambiental, y un adecuado manejo sanitario. Tal vez más importante en las condiciones actuales sea la optimización de variables externas. Entre las más importantes que ahora afectan la producción de leche están el acceso al crédito, especialmente de capitalización; la existencia de canales eficientes de acopio, transformación, y comercialización; la accesibilidad a la información de mercado; infraestructua vial adecuada; y una política general de protección a la actividad pecuaria en Ucayali. 
Cuadro 2. Investigación proyectada por el IVITA dirigida a resolver las principales limitantes de orden alimentario que afectan la producción de leche en Ucayali

\begin{tabular}{cc}
\hline Limitante & Investigación proyectada \\
\hline
\end{tabular}

Germoplasma forrajero de Introducción, evaluación y desarrollo de baja digestibilidad germoplasma preseleccionado por alta calidad nutritiva.

Germoplasma forrajero con Introducción, evaluación y desarrollo de baja tolerancia al estrés de germoplasma preseleccionado por tolerancia a sequía sequía.

Disminución de la oferta Manejo agronómico de cratilia para forrajera en época seca producción en época seca.

Potencial de cratilia en sistemas de corte y acarreo para suplementación en época seca.

No se usan estrategias de Estrategias de alimentación de terneros con alimentación para mejorar el amamantamiento restringido.

comportamiento reproductivo Adecuación de la técnica de rangos de condición corporal en el manejo reproductivo.

Disponibilidad de forraje de mejor calidad por mejores opciones de germoplasma y/o manejo más eficiente del pastoreo
Incremento de la productividad global del hato por una mejor estrategia de alimentación para el comportamiento reproductivo
Reducción de la caída de producción en época seca por el uso de pasturas tolerantes a la sequía y/o opciones de suplementación estacional con leguminosas arbustivas

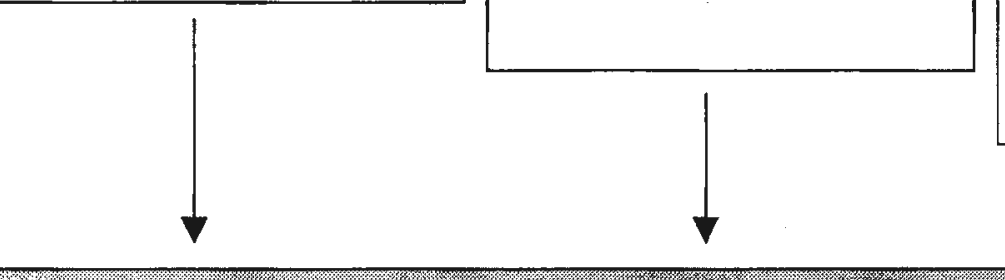

PRODUCTORES PRODUCEN MÁS LECHE Y CON MAYOR EFICIENCIA

Figura 2. Componentes del impacto de la investigación alimentaria en producción bovina con ordeño a nivel de productores en Ucayali. 
Cuadro 3. Principales resultados obtenidos y esperados como producto de la investigación alimentaria en la producción bovina con ordeño en Ucayali.

Limitante

Resultados obtenidos y esperados

Germoplasma forrajero de baja digestibilidad

Escasa adopción de leguminosas

Deficiente manejo del pastoreo

Germoplasma forrajero con baja tolerancia al estrés de sequía

Disminución de la oferta forrajera en época seca

No se usan sistemas de alimentación para mejorar el comportamiento reproductivo
- Dos o tres nuevas opciones de gramíneas de alta calidad nutritiva (a obtenerse).

- Densidades óptimas de siembra y fertilización adecuada para el establecimiento de $C$. Macrocarpum (obtenido).

- La urea en la leche es un buen indicador metabólico del consumo de leguminosa (obtenido).

- La introducción de leguminosas al pastoreo produce incrementos marginales $(7 \%)$ en la producción de leche (obtenido).

- Edades de rebrote adecuadas para el pastoreo rotativo de B. Dictyoneura (a obtenerse).

- Dos o tres nuevas opciones de gramíneas y leguminosas tolerantes a la sequía (a obtenerse).

- La suplementación convencional estacional mantiene la producción de leche durante el año (obtenido).

- Manejo de corte y fertilización de cratilia para optimizar productividad en época seca (a obtenerse).

- Potencial de cratilia como suplemento alternativo en época seca (a obtenerse).

- La suplementación convencional estacional reduce en cuatro meses el período para alcanzar peso de empadre de vaquillas de reemplazo (obtenido).

- La suplementación mineral con $60 \mathrm{~g} / 400 \mathrm{~kg}$ de peso vivo-semana es la más adecuada (obtenido).

- Rangos de condición corporal en uso por los productores para el manejo reproductivo del hato (a obtenerse). 


\section{Witerittma Ctadh}

1. CONTRADROGAS. 1998. Producción animal en áreas de desarrollo alternativo. Memoria de Taller. USAID/PERUWinrock-Acción Agraria-CONTRADROGAS. Pachacamac 12-13 de Junio de 1998.

2. De la Torre, M., C. Reyes y M. Ara. 1998. Suplementación permanente o estacional para vaquillas de reemplazo en pasturas tropicales. Rev Inv Pec IVITA (Perú) 9(1):59-66.

3. IVITA. 1972. Investigaciones pecuarias del IVITA en el trópico. UNMSMIVITA Bol Div 9.

4. Oficina de Información Agraria. 1997. Boletín informativo 1996-97. Dirección Regional de Agricultura de Ucayali.

5. Pizarro, E. y L. Coradin (ed.). 1995.
Potencial del género Cratylia como leguminosa forrajera. P. 98-106. En Memorias del Taller de Trabajo sobre Cratylia. 19-20 de Julio de 1995. Brasilia, DF. Brasil.

6. Riesco, A. 1997. Consumo de leche en la selva? Consorcio para el Desarrollo Sostenible de Ucayali. Bol Inf 3.

7. Toledo, J.M. y R. Schultze-Kraft. 1982. Metodología para la evaluación agronómica de pastos tropicales. p. 91110. En J.M. Toledo (ed.) Manual para la evaluación agronómica: Red Internacional de Evaluación de Pastos Tropicales. CIAT. Cali, Colombia.

8. TROPILECHE. 1998. Taller Internacional sobre Actividades de TROPILECHE. Memoria de Taller. Consorcio TROPILECHE. Atenas, Costa Rica 24-26 de Febrero de 1998. 